\title{
2-Methoxyestradiol Plasma Levels Are Associated With Clinical Severity Indices and Biomarkers of Preeclampsia
}

Reproductive Sciences

2015, Vol. 22(2) 198-206

(C) The Author(s) 2014

Reprints and permission:

sagepub.com/journalsPermissions.nav DOI: I0.1 I77/I9337|9||45377|6

rs.sagepub.com

(S)AGE

\author{
Miriam Pertegal, MD, MPH', Francisco J. Fenoy, MD, PhD', \\ Barbara Bonacasa, $\mathbf{P h D}^{2}$, Jaime Mendiola, $\mathrm{PhD}, \mathrm{MPH}^{3}$, \\ Juan L. Delgado, MD, PhD', Moises Hernández, $\mathrm{MSc}^{2}$, \\ Miguel G. Salom, MD, $\mathrm{PhD}^{2}$, Vicente Bosch, MD, $\mathrm{PhD}^{3,4}$, \\ and Isabel Hernández, MD, PhD ${ }^{2}$
}

\begin{abstract}
We investigated whether clinical severity indices and biomarkers for preeclampsia (PE) are associated with low plasmatic 2-methoxyestradiol (2ME) in the third trimester of gestation. Blood was collected from 53 women with PE and 73 control pregnant women before parturition. The concentration of $2 \mathrm{ME}$ was significantly higher in controls than in patients with PE (2906.43 \pm $200.69 \mathrm{pg} / \mathrm{mL}$ vs $1818.41 \pm 189.25 \mathrm{pg} / \mathrm{mL}$ ). The risk of PE decreased as $2 \mathrm{ME}$ levels increased. The $2 \mathrm{ME}$ values were negatively correlated with systolic peak arterial pressure and proteinuria in PE. Additionally, those women with PE with lower $2 \mathrm{ME}$ had a more serious clinical situation and needed a more aggressive therapy. Finally, 2ME levels (in patients with PE and total population) were significantly correlated with concentrations of soluble fms-like tyrosine kinase I and placental growth factor . Summarizing, patients with PE had lower 2ME levels that were correlated with different clinical indices and biomarkers of severity, indicating that $2 \mathrm{ME}$ could be taken into account for the clinical management of this syndrome.
\end{abstract}

\section{Keywords}

pregnancy, estrogen, catechol-O-methyltransferase, sFlt-I, PIGF

\section{Introduction}

Preeclampsia (PE) affects $2 \%$ to $8 \%$ of pregnancies worldwide, ${ }^{1}$ being a leading cause of preterm birth and causing important neonatal morbidity and mortality in the developed world. Furthermore, PE is still a major cause of death during pregnancy around the world, accounting for $10 \%$ to $15 \%$ of direct maternal deaths overall. ${ }^{2}$

In the last years, many efforts have been devoted to clarify the pathophysiology of this syndrome. However, the mechanisms underlying its pathogenesis remain unclear, and the delivery of the placenta is still the only definitive treatment. Placental ischemia and angiogenic imbalance seem to be involved in the pathophysiology of PE because a poor placentation appears to cause a hypoxic condition ${ }^{3}$ that increases the levels of proangiogenic proteins such as vascular endothelial growth factor (VEGF), VEGF receptor 1, and placental growth factor (PlGF). ${ }^{4}$ Recent research has been focused on the role of catechol-O-methyltransferase (COMT) and its product 2-methoxyestradiol (2ME), a naturally metabolite of estradiol. ${ }^{5}$ Serum $2 \mathrm{ME}$ rises steadily during the progress of pregnancy ${ }^{6}$ and reaches a plateau during the last trimester. ${ }^{7} \mathrm{~A}$ role for $2 \mathrm{ME}$ in the pathophysiology of PE seems likely because this estrogen appears to be a key factor in maintaining placental homeostasis necessary for appropriate cytotrophoblast invasion of the maternal decidua, ${ }^{8}$ and it has been demonstrated that COMT knockout mice $\left(\mathrm{COMT}^{-/-}\right)$exhibit a PE-like phenotype that is prevented by the administration of $2 \mathrm{ME}^{9}$

Many beneficial cardiovascular effects have been attributed to 2ME. 2-Methoxyestradiol inhibits injury-induced neointima formation and blocks abnormal cellular growth in vascular smooth muscle. ${ }^{10}$ 2-Methoxyestradiol also reduces atherosclerotic lesion

\footnotetext{
' Department of Gynaecology and Obstetrics, "Virgen de la Arrixaca" Clinical Universitary Hospital, El Palmar (Murcia), Spain

${ }^{2}$ Department of Physiology, School of Medicine, University of Murcia, Espinardo (Murcia) Spain

${ }^{3}$ Division of Preventive Medicine and Public Health, Department of Health and Social Sciences, School of Medicine, University of Murcia, Espinardo (Murcia), Spain

${ }^{4}$ Department of Pediatrics, "Virgen de la Arrixaca" Clinical Universitary Hospital, El Palmar (Murcia), Spain.
}

\section{Corresponding Author:}

Francisco J. Fenoy, Department of Physiology, School of Medicine, University of Murcia, 30100 Campus Universitario de Espinardo, Murcia 30100, Spain.

Email: fjfenoy@um.es 
Table I. Clinical Characteristics of the Population Sample.

\begin{tabular}{|c|c|c|c|}
\hline & Preeclampsia $(n=53)$ & Control $(n=73)$ & Total $(\mathrm{N}=126)$ \\
\hline Maternal age, years & $31.47 \pm 0.75$ & $32.19 \pm 0.65$ & $31.89 \pm 0.49$ \\
\hline Body mass index, $\mathrm{kg} / \mathrm{m}^{2}$ & $26.85 \pm 0.74^{\mathrm{a}}$ & $24.96 \pm 0.48$ & $25.74 \pm 0.42$ \\
\hline \multicolumn{4}{|l|}{ Smoking, $\mathrm{n}$} \\
\hline Nonsmokers + exsmokers & 47 & 55 & 102 \\
\hline Smokers & 5 & 16 & 21 \\
\hline Gestational diabetes, $\mathrm{n}$ & 6 & 4 & 10 \\
\hline Admission systolic blood pressure, $\mathrm{mm} \mathrm{Hg}$ & $156.04 \pm 1.76^{b}$ & $112.22 \pm 1.33$ & $130.65 \pm 2.21$ \\
\hline Admission diastolic blood pressure, $\mathrm{mm} \mathrm{Hg}$ & $96.36 \pm 1.36^{\mathrm{b}}$ & $61.78 \pm 1.06$ & $76.33 \pm 1.74$ \\
\hline Proteinuria, $\mathrm{n}$ & 53 & 0 & 53 \\
\hline Gestational days at delivery, days & $242.7 \pm 3.96^{\mathrm{b}}$ & $282.12 \pm 1.02$ & $265.54 \pm 2.47$ \\
\hline Birth weight, g & $2139.79 \pm 129.93^{b}$ & $3591.88 \pm 53.78$ & $2979.82 \pm 89.71$ \\
\hline
\end{tabular}

a $P<.05$ compared to control pregnancy.

${ }^{\mathrm{b}} P<.0$ l compared to control pregnancy.

formation ${ }^{11}$ and improves vascular endothelial cell function decreasing the release of endothelin $1^{12}$ and inducing the production of vasoprotective substances such as prostacyclin. ${ }^{13}$ Also, 2ME has a vasodilatory influence by increasing endothelium-dependent nitric oxide (NO) production, ${ }^{14,15}$ and given its powerful antioxidant properties, ${ }^{16,12}$ it may potentiate NO bioavailability by preventing its oxidation. ${ }^{5}$ However, there are no studies showing any association between levels of $2 \mathrm{ME}$ and clinical features in patients with PE.

Taken together, the available evidence points at COMTgenerated $2 \mathrm{ME}$ as a central factor in the pathophysiology of PE. ${ }^{9}$ Therefore, the aim of the present study was to provide clinical evidence that $2 \mathrm{ME}$ is decreased in PE, and its level correlates with the severity of the disease in humans.

\section{Methods}

This is an observational, analytical, and prospective casecontrol study. A total of 126 pregnant women (53 PE and 73 control, normal pregnancies) were included in the present study prospectively from November 2009 to January 2012 at the Hospital Clínico Universitario Virgen de la Arrixaca (HCUVA) in Murcia, Spain. Preeclampsia was defined as blood pressure (BP) elevation with proteinuria $(300 \mathrm{mg}$ or more per 24-hour period). If 24-hour urine collection was not available then proteinuria was defined as 2 or more "+" obtained using a dipstick test. Blood pressure elevation was defined as an arterial pressure e $\geq 140 / 90 \mathrm{~mm} \mathrm{Hg}$ on at least 2 occasions separated by a minimum of 6 hours after the 20th week of gestation in women known to be normotensive before pregnancy and also during the first weeks of gestation. Controls were women with uncomplicated pregnancies that lasted 37 weeks (or 259 days) or more. All of the participants had singleton pregnancies and were of white race. None of the participants had a history of chronic hypertension, renal or infectious diseases, or pregestational or gestational insulindependent diabetes.
The ethic committee of clinical investigation of the HUVA and the bioethics committee of the Universidad de Murcia approved the research protocol, including the consent form. Written informed consent was obtained from each participant, and the study was conducted in accordance with the Declaration of Helsinki.

In this study, mother blood was drawn by routine forearm venipuncture at the time of admission. In patients with PE, blood was extracted when it was decided to end the pregnancy, the same day or the day before delivery. In controls, blood was drawn during a routine consultation of 1 to 7 days before parturition. Gestational age at delivery for both groups is presented in Table 1. Blood was collected in a dry tube for serum analysis and in a tube containing EDTA for plasma analysis. Samples were immediately centrifuged at $2500 \mathrm{rpm}$ for 7 minutes and then aliquoted under sterile conditions and stored at $-80^{\circ} \mathrm{C}$ until use.

Serum levels of maternal PIGF and soluble fms-like tyrosine kinase 1 (sFlt-1) were measured by an electrochemiluminescence immunoassay (Roche Diagnostics, Mannheim, Germany, No 05144671 and 05109523 , respectively) on the automated Roche immunoanalyzer COBAS e 411 (Roche Diagnostics, Germany) following the manufacturer's recommendations. Measurement of plasma 2-ME was performed by enzymoimmunoassay (Cayman Chemical Company, Michigan, No 582261). The extraction of $2 \mathrm{ME}$ from plasma was performed by a double solvent procedure (ethanol and methylene chloride) following the manufacturer's recommendations. Briefly, ethanol $(1.5 \mathrm{~mL})$ was added to plasma samples $(0.5$ $\mathrm{mL}$, in duplicate), vortexed, and centrifuged at $1250 \mathrm{~g}$ for 10 minutes. Supernatants were evaporated at $30^{\circ} \mathrm{C}$ under vacuum overnight, reconstituted in $0.5 \mathrm{~mL}$ of ultrapure water, and extracted 3 times with methylene chloride (mixed with $2 \mathrm{~mL}$ of methylene chloride, vortexed, and the methylene chloride layer transferred to a clean tube). Finally, methylene chloride was evaporated at $30^{\circ} \mathrm{C}$ under vacuum overnight, the dry sample reconstituted in assay buffer, and measured. The specificity 
of the 2ME enzyme immunosorbent assay antiserum available from Cayman does not include the cross-reactivity to $4 \mathrm{ME}$. However, this is unlikely to be a significant interference because it has been reported that $4 \mathrm{ME}$ plasma levels are very low during pregnancy and unaffected by $\mathrm{PE}$ $(\approx 20-35 \mathrm{pg} / \mathrm{mL}) .{ }^{17}$ The other biochemical parameters were taken from the clinical chart of the patients as part of routine tests done in the clinical laboratory of the HCUVA. Some missing data were caused by logistical problems and the prospective nature of the study. In addition, for some participants, only small amounts of blood were available, resulting in different numbers for the separate analyses.

\section{Statistical Analyses}

All data are presented as mean \pm 1 standard error of the mean unless otherwise indicated. Some variables were logtransformed to obtain normal distributions for subsequent parametrical statistical analysis. Student $t$ test was used to compare the means of baseline characteristics and different biomarkers between control and PE groups. The chi-square $\left(\lambda^{2}\right)$ test was employed to make case and control comparisons for categorical variables. The association between $2 \mathrm{ME}$ and levels of different parameters was evaluated using Pearson correlation coefficient. To compare frequencies of population distribution into each quartile of $2 \mathrm{ME}$, a nonparametric (one-sample $\lambda^{2}$ ) test was employed. Multiple logistic regression analysis was used to estimate the adjusted odds ratio (adjOR) of $\mathrm{PE}$ according to mean levels and quartiles of $2 \mathrm{ME}$. In order to explore the adjusted associations for a continuous outcome variable, multiple linear regressions were also used. Analysis of covariance (ANCOVA) followed by a post hoc Bonferroni test was employed to compare $2 \mathrm{ME}$ levels between different antihypertensive treatment groups. The following covariates were taken into account in all the adjusted analyses: smoking habit (smoker during pregnancy, never smoked during pregnancy, or smoker that ceased at the beginning of the pregnancy), body mass index (BMI), maternal age, and nulliparity (yes/no). A $P$ value $<.05$ (2-tailed test) was considered to be significant. The statistical software package SPSS v19.0 (IBM-SPSS Inc, Chicago, Illinois) was used for all the analyses.

\section{Results}

Clinical characteristics of the 126 women are presented in Table 1. As we expected, based on the definition, women with PE collectively had proteinuria and significant higher systolic and diastolic BPs than that of normal pregnant women. The BMI was significantly greater in women with PE, and this group had a significantly higher rate of nulliparity. The number of smokers was higher in controls than in women with $\mathrm{PE}$, but the difference was not significant $(P=.06)$. In addition, $2 \mathrm{ME}$ values were not significantly different between smokers and nonsmokers within the control population $(2563.66 \pm 374.64$ vs $2886.03 \pm 255.48 \mathrm{pg} /$ $\mathrm{mL} ; P=.6)$. The gestational age at delivery for women with PE was significantly lower than that in the group of women with

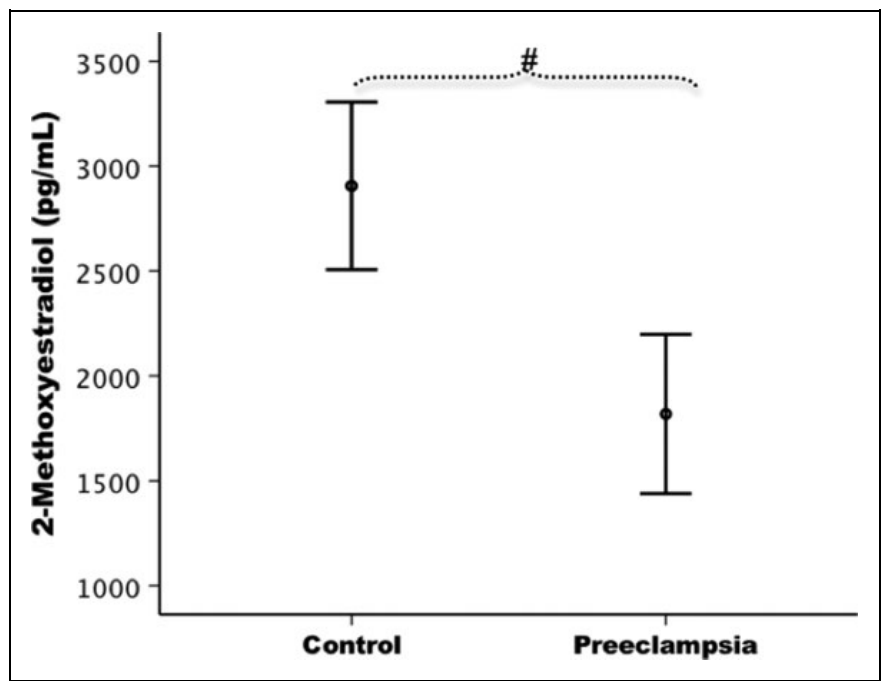

Figure I. Levels of 2-methoxyestradiol in maternal plasma.The graph shows the mean and $95 \%$ confidence interval of 2-methoxyestradiol in maternal plasma in the population with preeclampsia and control population samples. ${ }^{\#} p<.001$.

uncomplicated pregnancies, and the birth weight was significantly greater in this control group. There were no statistical differences between controls and PE in maternal age, use of assisted reproductive techniques, including oocyte donation, and gestational diabetes controlled with diet.

Control individuals $(n=66)$ had significantly higher $2 \mathrm{ME}$ plasma levels than women with $\mathrm{PE}(\mathrm{n}=50 ; 2906.43 \pm$ $200.69 \mathrm{pg} / \mathrm{mL}$ vs $1818.41 \pm 189.25 \mathrm{pg} / \mathrm{mL} ; P<.001$; Figure 1). Additional multiple logistic regression analysis was performed to include potential confounders (smoking habit, BMI, maternal age, and nulliparity). Increased plasmatic $2 \mathrm{ME}$ was observed to have a significant effect $(P<.001)$ and was associated with a decreased risk of PE. The adjOR was $.05(95 \%$ confidence interval [CI]: 0.01-0.25; $\mathrm{n}=113$ ).

Plasma levels of $2 \mathrm{ME}$ were used to separate the population samples into quartiles resulting in an ordered progression of frequency distribution in both women with PE and control women. When the distribution of frequencies of the population samples was compared, we observed a higher than expected number of patients having $\mathrm{PE}$ with levels of $2 \mathrm{ME}$ in the first quartile (Figure 2), while the number of women with PE was lower than expected for third and fourth quartiles. The results of a logistic regression model show a decreasing trend in the risk of $\mathrm{PE}$ as $2 \mathrm{ME}$ levels increase $(P$ [trend] $=.001)$. In fact, protection is triggered when levels exceed the median value (1988.24 pg/mL). The adjOR for the third and the 4th quartiles compared with the first quartile was 0.129 (95\% CI: 0.04-0.45; $P<.001)$ and 0.157 (95\% CI: 0.05-0.55; $P<.004)$, respectively (Table 2).

As shown in Figure 3, we investigated the connection between levels of $2 \mathrm{ME}$ and the 2 components of the definition of PE, arterial hypertension (systolic and diastolic peak arterial pressure), and proteinuria in 24-hour urine samples. After adjusting, we found that the $2 \mathrm{ME}$ values were significantly and 


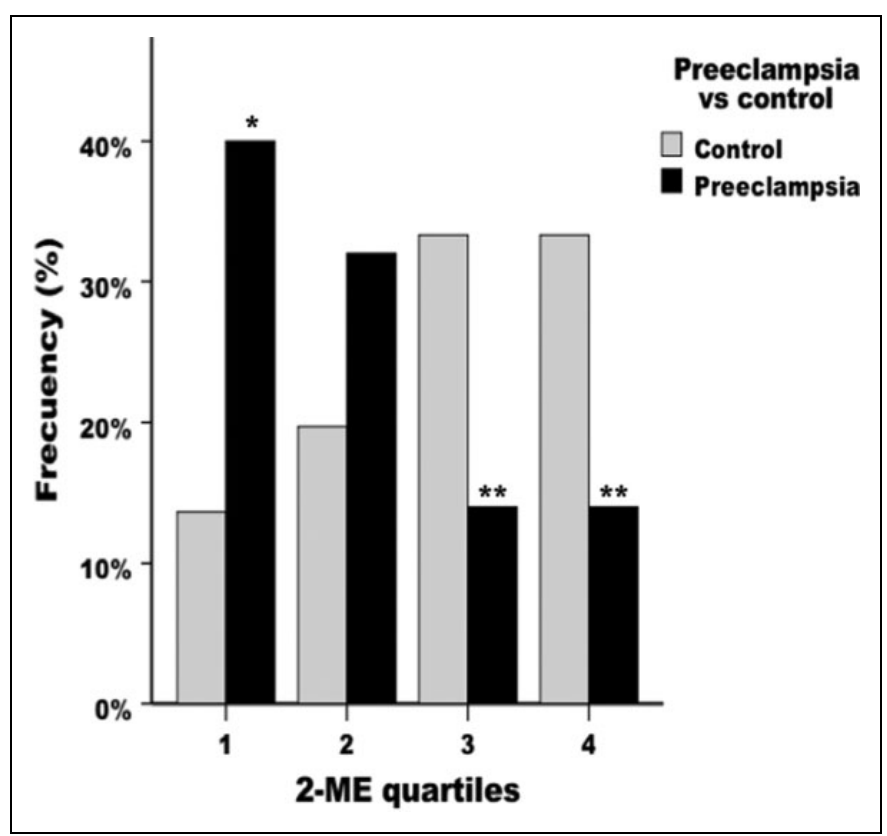

Figure 2. Frequency distribution of 2-methoxyestradiol. Each bar represents the percentage of the total population in each quartile of $2 \mathrm{ME}$. $* P<.05$ compared to control pregnancies. $* * P<.01$ compared to control pregnancies.

negatively correlated with both systolic peak BP and proteinuria. There was no correlation between $2 \mathrm{ME}$ and diastolic peak BP $(P=.66$; Table 3$)$.

In order to test whether plasma levels of $2 \mathrm{ME}$ were associated with the severity of arterial hypertension in women with $\mathrm{PE}$, we divided our patients as a function of the intensity of the therapy needed to control hypertension: no treatment, oral treatment, and intravenous treatment. The change in therapy was conducted depending on whether the patients kept their $\mathrm{BP}$ in levels near 140/90 mm Hg and under 160/100 mm Hg with their respective treatments. The patients with $\mathrm{PE}$ who needed more aggressive therapy to control arterial pressure had lower plasmatic levels of 2ME. Thus, those who did not need any treatment $(\mathrm{n}=8)$ showed levels of $2598.15 \pm 608.97$ $\mathrm{pg} / \mathrm{mL}$; women who only needed oral treatment $(\mathrm{n}=22)$ presented levels of $2174.99 \pm 308.27 \mathrm{pg} / \mathrm{mL}$; and women who needed intravenous treatment ( \pm oral treatment; $\mathrm{n}=20$ ) had levels of $1114.27 \pm 129.25 \mathrm{pg} / \mathrm{mL}$. After adjusting for confounding factors (ANCOVA), women with PE who needed intravenous treatment to control their BP had significantly lower plasma levels of $2 \mathrm{ME}$ than the other 2 groups, respectively $(P=.013 \mathrm{vs}$ the untreated group and $P=.016 \mathrm{vs}$ the group with oral treatment; Figure 4).

With the purpose of delving into aspects of clinical severity of $\mathrm{PE}$, we correlated $2 \mathrm{ME}$ levels as a function of the presence of clinical signs and symptoms of severity: cerebral or visual disturbances (headache, blurred vision, photopsias, and scotomata), oliguria $(<0.5 \mathrm{~mL} / \mathrm{kg} / \mathrm{h})$, or epigastric or right upper-quadrant abdominal pain. The 14 women with PE having clinical severity signs had lower levels of $2 \mathrm{ME}(1296.18 \pm 272.63 \mathrm{pg} / \mathrm{mL})$ than the 36 women with PE who did not $(2021.50 \pm 233.87 \mathrm{pg} / \mathrm{mL}$,
$P=.05$ ). Thus, higher levels of $2 \mathrm{ME}$ seem to have a significant effect associated with a decreased risk of severe PE (adjOR 0.115 ; 95\% CI: $0.014-0.964 ; P=.046 ; \mathrm{n}=49$ ).

Regarding different analytical biomarkers of renal and liver function related to the severity of PE, as shown in Table 4, women with PE had a significantly higher levels of serum uric acid, creatinine, lactate dehydrogenase (LDH), aspartate aminotransferase (AST), and alanine aminotransferase (ALT) than control pregnant women. Nevertheless, the creatinine mean levels in the population with PE were within the normal range $(0.5-0.9 \mathrm{mg} / \mathrm{dL})$, and mean levels of AST and ALT were at the upper limit of normality (5-31 UI/L). There was no difference in platelet counts between both the groups. We also explored the association between those biomarkers and $2 \mathrm{ME}$ levels. The plasmatic levels of $2 \mathrm{ME}$ in the population with $\mathrm{PE}$ were significantly and negatively correlated with serum levels of uric acid, creatinine, and LDH. In addition, the $2 \mathrm{ME}$ levels were significantly and positively associated with platelet counts. There was no significant correlation with AST and ALT (Table 3).

Given the well-known dependence of birth weight on infant outcomes, we also analyzed the association between birth weight and $2 \mathrm{ME}$. The plasma $2 \mathrm{ME}$ levels of the population with PE $(n=49)$ were significantly and positively associated with birth weight $\left(\mathrm{B}=1.5 \cdot 10^{-4} ; 95 \% \mathrm{CI}: 5.6 \cdot 10^{-5}\right.$ $\left.2.44 \cdot 10^{-4} ; P=.002\right)$. The slope of this relationship was about $1 \mathrm{pg} \mathrm{mL}^{-1} 2 \mathrm{ME} / 1 \mathrm{~g}$ of body weight of the newborn (Table 3 ).

Regarding the widely studied angiogenic and antiangiogenic factors involved in the pathogenesis of PE, we investigated the serum levels of these factors and its association with 2ME. Serum concentration of sFlt1 was significantly increased (4-fold) in women with PE compared with control pregnant women $(P<.001)$. Serum PlGF concentration was decreased 4-fold in the pregnant women with PE compared with uncomplicated pregnancies $(P<.001$; Table 4$)$.

The plasmatic levels of $2 \mathrm{ME}$ in the total population sample (preeclamptic + controls) were significantly and negatively associated with serum levels of sFlt-1 and significantly and positively associated with serum levels of PIGF (Figure 5). Both correlations remained significant after adjusting for potential confounding factors $(\mathrm{n}=113 ; \mathrm{B}=-0.32 ; 95 \%$ CI: -0.48 to $-0.16 ; P<.001$ and $\mathrm{B}=0.34 ; 95 \% \mathrm{CI}: 0.20$ $0.47 ; P<.001$ respectively).

Finally, 2ME plasma levels in women with PE were also significantly correlated with sFlt-1 and PIGF (Figure 5). After adjustment, 2ME plasma levels remained significantly and negatively associated with sFlt1 levels $(\mathrm{B}=-0.54 ; 95 \% \mathrm{CI}$ : -0.91 to $-0.17 ; P=.006)$ and significantly and positively associated with PlGF levels (B $=0.48 ; 95 \%$ CI: 0.16-0.80; $P=.005$; Table 3 ).

\section{Discussion}

This report presents interesting and novel data about the relationship between $2 \mathrm{ME}$ plasma levels and a variety of clinical severity indices in women with PE. It was found that patients with PE had lower circulating levels of 2ME than women with uncomplicated pregnancies during the third trimester. In 
Table 2. Odds ratio (OR) and 95\% Confidence Interval (Cl) of Preeclampsia According to the Quartile of 2-Methoxyestradiol (2ME) Concentrations.

\begin{tabular}{lccc}
\hline Quartile & 2-ME, pg/mL & Unadjusted OR (95\% Cl) & Adjusted ${ }^{\mathrm{a} O R}(95 \% \mathrm{Cl})$ \\
\hline Quartile I & $<1204.5 \mathrm{I}$ & 1.0 (reference) $^{-}$ & $1.0($ reference) \\
Quartile 2 & $1204.52-1988.245$ & $0.55(0.19-1.62)$ & $0.78(0.24-2.53)$ \\
Quartile 3 & $1988.246-3230.123$ & $0.14(0.05-0.46)^{\mathrm{b}}$ & $0.13(0.04-0.45)^{\mathrm{b}}$ \\
Quartile 4 & $>3230.123$ & $0.14(0.05-0.46)^{\mathrm{b}}$ & $0.16(0.05-0.55)^{\mathrm{b}}$ \\
\hline
\end{tabular}

${ }^{a} \mathrm{OR}$ and $95 \% \mathrm{Cl}$ adjusted for maternal age, body mass index, smoking habit, and nulliparity.

${ }^{b} P<.01$ compared with first quartile.

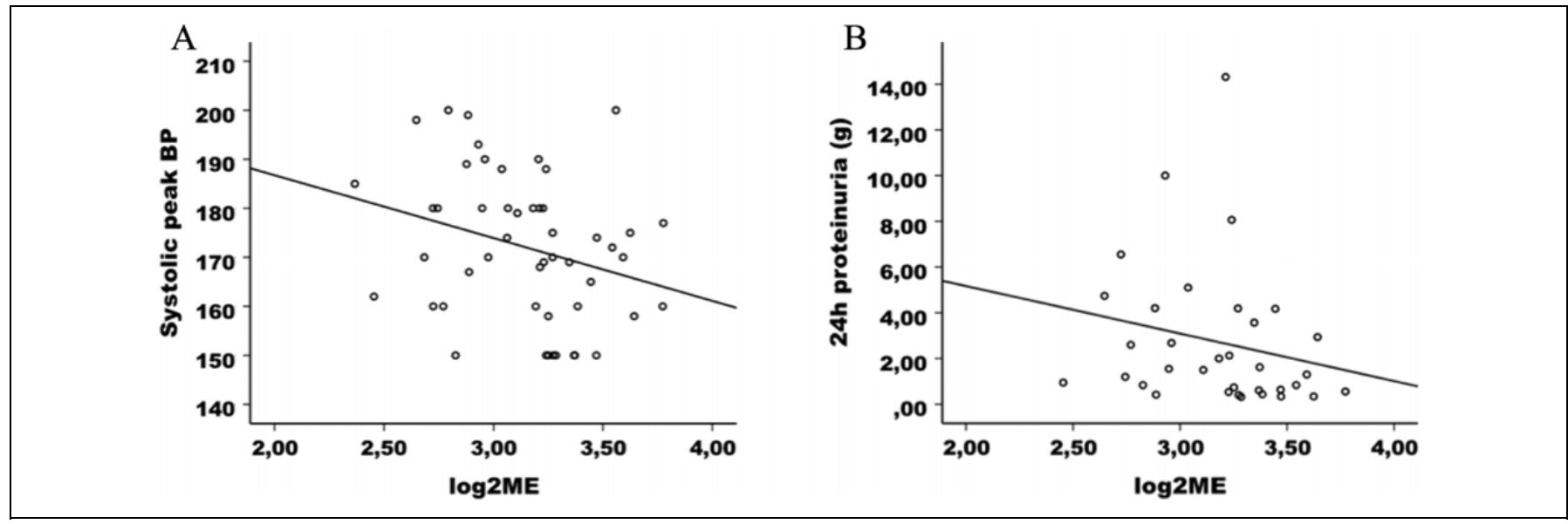

Figure 3. Correlation of 2-methoxyestradiol with blood pressure and proteinuria. A, Negative correlation in the preeclamptic population sample between maternal $2 M E$ levels and systolic peak blood pressure (BP; $n=50 ; r=-.283 ; P=.047)$. B, Negative correlation in the population with preeclampsia sample between maternal $2 M E$ levels and proteinuria $(n=34 ; r=-.212 ; P=.229)$.

Table 3. Correlations of 2-Methoxyestradiol ${ }^{\mathrm{b}}(\mathrm{pg} / \mathrm{mL})$ and Clinical and Biochemical Parameters in Population With Preeclampsia.

\begin{tabular}{|c|c|c|c|c|c|}
\hline \multirow[b]{2}{*}{ Parameter } & \multirow[b]{2}{*}{$\mathrm{n}$} & \multirow[b]{2}{*}{ Unadjusted $r(P)$} & \multirow[b]{2}{*}{$\mathrm{n}$} & \multicolumn{2}{|l|}{ Adjusted $^{\mathrm{a}}$} \\
\hline & & & & $\mathrm{B}(95 \% \mathrm{Cl})$ & $P$ \\
\hline Diastolic peak blood pressure, $\mathrm{mm} \mathrm{Hg}$ & 50 & $-.073(.617)$ & 49 & $-0.002(-0.012$ to 0.008$)$ & .656 \\
\hline Proteinuria, $g$ & 34 & $-.212(.229)$ & 34 & $-0.042(-0.078$ to -0.006$)$ & .024 \\
\hline Birth weight, $g$ & 50 & $.447(.001)$ & 49 & $1.50^{-4}\left(5.60 \cdot 10^{-5}\right.$ to $\left.2.44 \cdot 10^{-4}\right)$ & .002 \\
\hline Uric acid, mg/dL & 42 & $-.388(.011)$ & 41 & $-0.078(-0.146$ to -0.010$)$ & .026 \\
\hline Creatinine, $\mathrm{mg} / \mathrm{dL}$ & 37 & $-.297(.074)$ & 36 & $-0.496(-0.984$ to -0.009$)$ & .046 \\
\hline Lactate dehydrogenase (LDH), U/L & 44 & $-.392(.008)$ & 43 & $-0.001\left(-0.002\right.$ to $\left.-3.16 \cdot 10^{-4}\right)$ & .010 \\
\hline Platelet, $/ \mu \mathrm{L}$ & 50 & $.252(.078)$ & 49 & $1.16 \cdot 10^{-6}\left(7.64 \cdot 10^{-8}\right.$ to $\left.2.24 \cdot 10^{-6}\right)$ & .036 \\
\hline Aspartate aminotransferase (AST), ${ }^{b}$ UI/L & 47 & $.009(.954)$ & 46 & $-0.098(-0.474$ to 0.279$)$ & .603 \\
\hline Alanine aminotransferase $(A L T)^{b}, \mathrm{UI} / \mathrm{L}$ & 47 & $.196(.186)$ & 46 & $0.059(-0.227$ to 0.344$)$ & .681 \\
\hline
\end{tabular}

${ }^{a}$ Correlation adjusted for maternal age, body mass index, smoking habit and nulliparity.

${ }^{b}$ log transformation.

addition, women with PE having lower levels of $2 \mathrm{ME}$ had higher values of systolic peak arterial pressure and proteinuria and they also needed a more aggressive treatment to control their BP and manifested a more serious clinical situation. Finally, 2ME levels were significantly correlated (positively and negatively, respectively) with angiogenic (PlGF) and antiangiogenic (sFlt1) factors that are strongly associated with the pathogenesis of PE.
The awareness about the relationship between COMTderived 2ME and PE started with Barnea et al who found a lower placental COMT activity in patients with severe PE. ${ }^{18}$ Later, Kanasaki et $\mathrm{al}^{9}$ postulated a possible role for decreased production of $2 \mathrm{ME}$ in human $\mathrm{PE}$ as a result of lower activity or expression of placental COMT. They based this hypothesis on the fact that COMT knockout mice $\left(\mathrm{COMT}^{-/}\right)$exhibited a PE-like phenotype that was prevented by the administration 


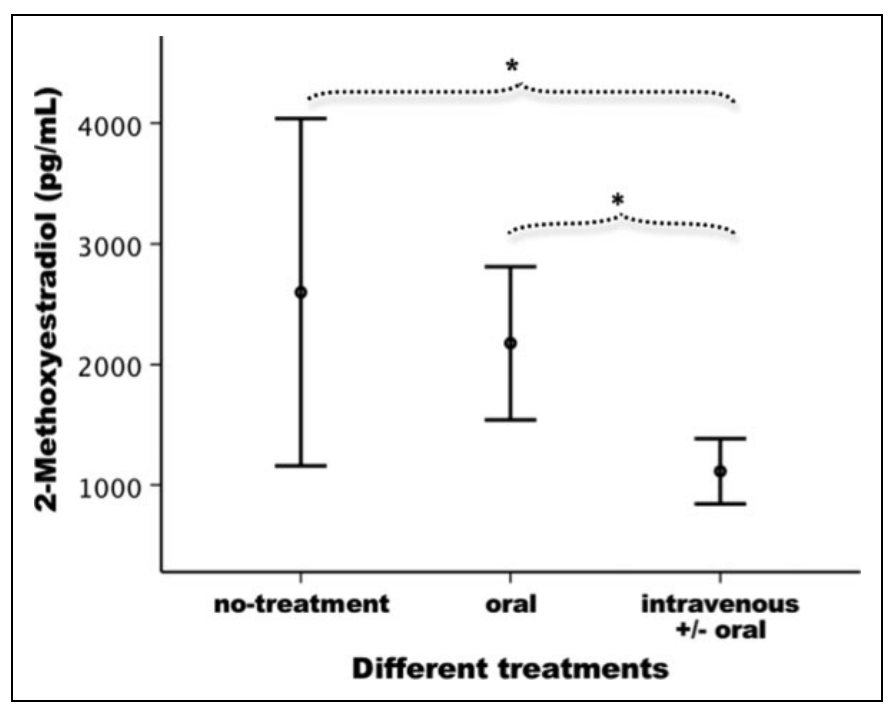

Figure 4. Values of 2-methoxyestradiol according to hypertension treatment. The graph shows the mean and $95 \%$ confidence interval of 2-methoxyestradiol levels in different groups of hypertension treatment in women with PE. *Significant difference between groups "no treatment" and "oral treatment" compared with "intravenous treatment" $(P=.016$ and $P=.013$, respectively).

of $2 \mathrm{ME}$. In the same line of thought, several studies have linked different genetic variations in the metabolic pathway of COMT, which could lead to a reduction in $2 \mathrm{ME}$ circulating levels, with increased risk of PE. ${ }^{19-21}$ In this regard, Sata et $\mathrm{al}^{22}$ found a gene polymorphism causing both low COMT activity and intrauterine fetal growth restriction, a syndrome that seems to share a common pathogenic basis with PE. ${ }^{23}$ Finally, Lee et $\mathrm{al}^{8}$ postulated the importance of $2 \mathrm{ME}$ (under hypoxic conditions) in achieving the appropriate cytotrophoblast invasion that allows for adequate fetal oxygenation and placental vascular development during pregnancy. Despite all these developments focused on the role of COMT and its metabolite $2 \mathrm{ME}$ in relation to the pathogenesis of PE, the 2ME levels have only been measured in 2 previous studies with a limited number of pregnant women with $\mathrm{PE},{ }^{9,17}$ hence the importance of our findings.

The present study shows values of $2 \mathrm{ME}$ in our control population sample in the same range compared to those reported previously (measured by immunoassay) in the literature for pregnant women in the third trimester ${ }^{6,7}$; similar results have also been found more recently in normal pregnancies using gas chromatography $^{24}$ or liquid chromatography ${ }^{9,17}$ followed by mass spectrometry. In addition, women with PE had lower levels of circulating $2 \mathrm{ME}$ than the control pregnant group, also in agreement with the literature. ${ }^{9,17}$ More importantly, we also show that the risk of PE increased especially as 2ME levels were approaching the first quartile of the variable distribution. In addition, women with PE who needed intravenous treatment to control arterial hypertension had significantly lower plasma levels of $2 \mathrm{ME}$ than all others. Taken together, those results indicate that $2 \mathrm{ME}$ could be used to predict the severity of PE.

The reduced 2ME levels measured in women with PE were associated with a wide range of severity of clinical features of the disease. For this reason, this group of patients provided a favorable opportunity to examine the relationships among the $2 \mathrm{ME}$ levels and BP, urinary protein, angiogenic and antiangiogenic factors, and the severity clinical indices of the disease. The present study establishes for the first time a clear correlation between $2 \mathrm{ME}$ levels and systolic arterial pressure in human $\mathrm{PE}$, and a more aggressive therapy was necessary to control the hypertension in women with lower levels of this estrogen metabolite. These findings are consistent with the results of Kanasaki's group, showing a significant increase in systolic $\mathrm{BP}$ in $\mathrm{COMT}^{-/-}$mice during pregnancy, effect that was prevented by exogenous administration of 2ME. An involvement of $2 \mathrm{ME}$ in the control of vascular tone seems likely because our group has previously demonstrated that $2 \mathrm{ME}$ decreases arterial pressure in both male and ovariectomized female spontaneously hypertensive rats. ${ }^{25}$ This beneficial effect seems to be related to increased endothelial NO bioavailability, which may be a result of a potentiation of $\mathrm{NO}$ release ${ }^{15}$ as well as a reduction in NO oxidation. ${ }^{26}$ This rise in NO bioavailability appears to oppose vascular smooth muscle contraction and reduce the effect of vasoconstrictors. ${ }^{14}$ During normal pregnancy, this vasorelaxing role of $2 \mathrm{ME}$ may become more important, given the need to accommodate the increased blood volume and cardiac output. Taken together, the available evidence strongly suggests that increasing amounts of $2 \mathrm{ME}$ are necessary to ensure high enough vascular NO levels essential for maintaining a reduced gestational vascular resistance. This idea is compatible with a recent report from our group showing that pharmacological inhibition of COMT activity during pregnancy causes arterial hypertension and endothelial dysfunction due to reduced NO bioavailability, ${ }^{26}$ supporting the idea that $2 \mathrm{ME}$ is a key factor in maintaining vascular endothelial health in normal pregnancy. Furthermore, in those experiments the presence of tempol improves vascular reactivity and NO release, suggesting the involvement of superoxide anion in endothelial dysfunction observed in this model of COMT inhibition.

Interestingly, in the present study plasma uric acid concentration was significantly higher in women PE than in controls, and $2 \mathrm{ME}$ levels in the preeclamptic population were significantly and inversely correlated with plasmatic uric acid levels. This may be noteworthy, because uric acid is the end product of purine metabolism, after a chemical reaction catalyzed by the enzyme xanthine oxidoreductase. ${ }^{27}$ Under hypoxic conditions, xanthine oxidase participates in this metabolic pathway resulting in superoxide anion production, which contributes to increase oxidative stress and also to reduce NO bioavailability, causing endothelial dysfunction. ${ }^{27}$ There is evidence in the literature indicating that uric acid levels correlate with $\mathrm{BP},{ }^{28}$ and also that high levels of uric acid are associated with greater severity of PE and adverse maternal and particularly fetal outcomes. ${ }^{29}$ Therefore, those studies indicate that an increased oxidative stress is present in $\mathrm{PE}$, and that a deficiency in COMT-derived 2ME may be involved in the increased free radical production that interferes with a normal placental and fetal development in PE. 
Table 4. Levels in Maternal Blood.

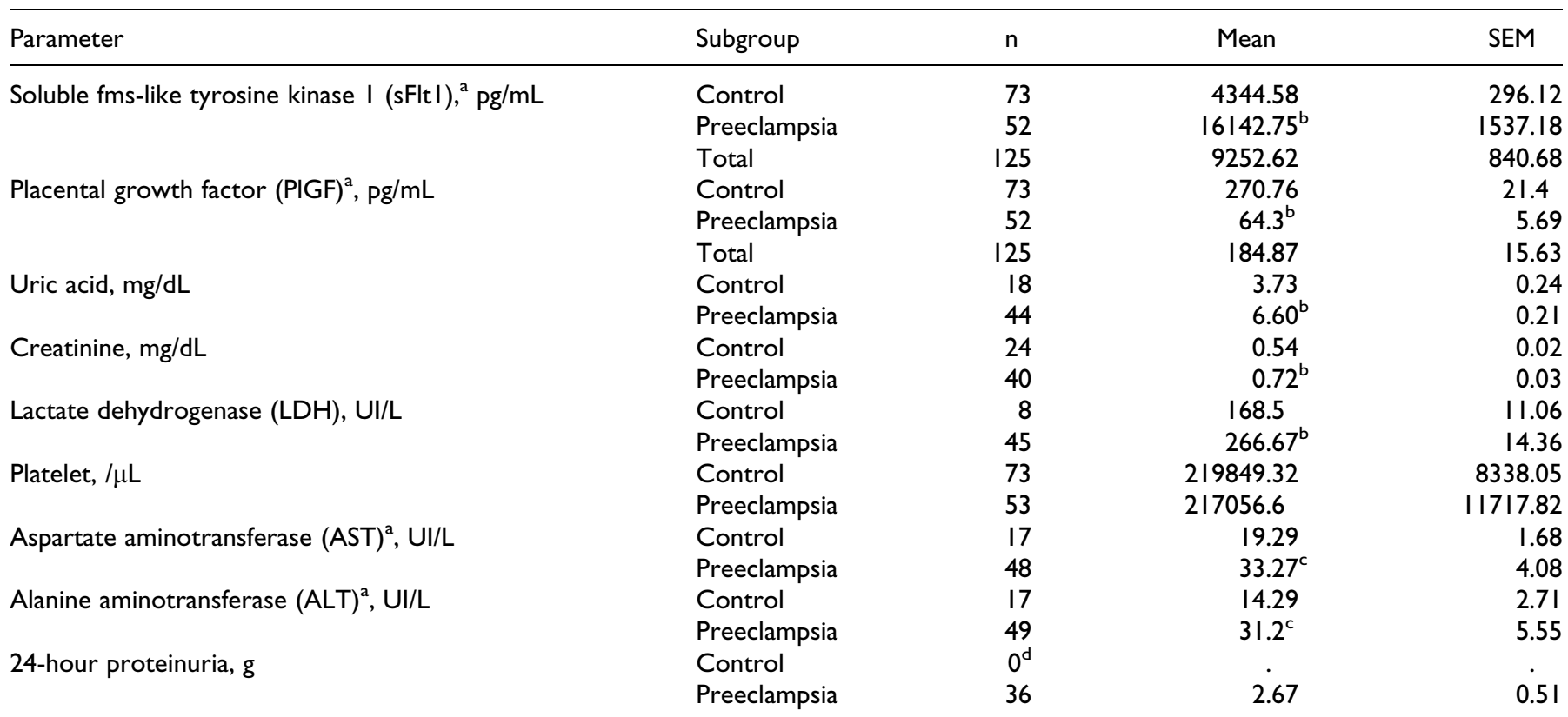

Abbreviation: SEM, standard error of the mean.

${ }^{a} \log$ transformation.

${ }^{b} P<0.00$ I compared with control pregnancy.

c $P<0.05$ compared with control pregnancy.

'Student's t couldn't be calculated because at least one of the groups was empty.

In the present study, an inverse correlation between $2 \mathrm{ME}$ concentration and both plasma creatinine and proteinuria was found in the preeclamptic population sample. These findings are consistent with a previous report showing that pregnant $\mathrm{COMT}^{-1-}$ mice developed renal endotheliosis with albuminuria, and also that this preeclamptic-like phenotype was avoided by exogenous $2 \mathrm{ME}$ administration. ${ }^{9}$ This protective glomerular action of $2 \mathrm{ME}$ is explained at the present by the inhibition of the abnormal growth of glomerular mesangial cells, ${ }^{30}$ the reduction in the deposit of extracellular matrix proteins such as collagen, and also by less inflammatory cell infiltration. ${ }^{31}$

In addition, descriptive statistics show that our population sample having PE had a greater BMI and a higher rate of nulliparity than controls. It is well known that both nulliparity $^{32}$ and obesity ${ }^{33}$ are risk factors for PE. Nevertheless, those factors are not invalidating our results because after adjusting for them, we found that the $2 \mathrm{ME}$ values were still significantly and negatively correlated with both systolic peak BP and proteinuria. Tobacco smoking has previously been found to have a protective effect as high as 30\% to $50 \%$ against $\mathrm{PE}^{34}$; however, in the present study, we found no significant differences between controls and patients with PE. Nevertheless, the possible protective effect of smoking is of particular interest because cytochrome P450 is induced by tobacco smoke in the placenta ${ }^{35}$ and this may result in higher hydroxylation of estradiol to 2-hydroxyestradiol and subsequent formation of $2 \mathrm{ME}$ in normal pregnancies. However, the data in the present study do not support a significant role for this mechanism because $2 \mathrm{ME}$ was actually lower (but not significantly different) in control smokers than in control nonsmoker pregnant women.

As expected, the levels of PlGF and sFlt1 in PE were significantly lower and higher, respectively, than that in normal pregnancies. The mean levels obtained for both biomarkers in our work were similar to those previously published in the literature. ${ }^{36}$ Finally, we demonstrated that plasma levels of 2ME were significantly correlated with levels of PIGF and sFlt1 (positive and negatively, respectively). The excess sFlt1 contributed to various features of the maternal syndrome such as placental hypoxia with placental insufficiency or renal glomerular endotheliosis. ${ }^{37}$ Thus, sFlt1 could be a factor linking placenta with maternal endothelial dysfunction. Placentas deficient in $2 \mathrm{ME}$ are not only prone to inflammation but can also have hypoxia and show increased nuclear levels of hypoxiainducible factor $1 \propto$ (HIF-1 $\alpha$ ), leading to an imbalance in angiogenic factors in the maternal serum. In support of this hypothesis, mice genetically deficient in $\operatorname{COMT}\left(\mathrm{COMT}^{-/-}\right)$ show an abnormal accumulation of HIF- $1 \alpha$ protein predominantly in the placenta, an effect that was reversed by the administration of 2ME. Moreover, $2 \mathrm{ME}$ ameliorated placental hypoxia by inhibition of HIF- $1 \alpha$ expression and precluded sFlt1 elevation. ${ }^{9}$ Thus, the available evidence indicates that placental COMT-generated 2ME not only protects against generalized endothelial dysfunction but also suppresses the placental synthesis and release of sFlt1.

Therefore, taking together the previous results and the data in the present study, showing that the angiogenic imbalance present in women with PE is associated with decreased 2ME plasma 

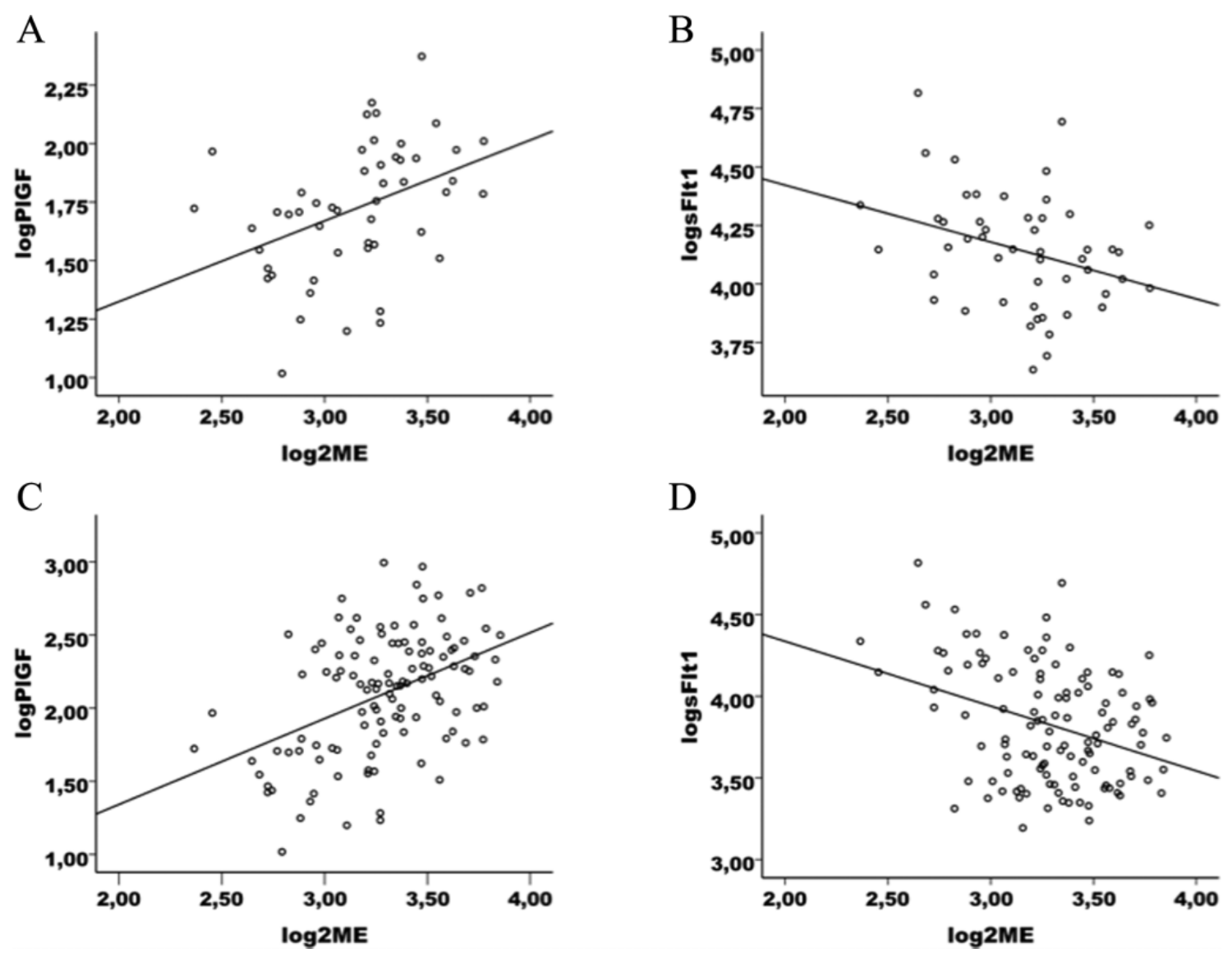

Figure 5. Correlation of 2-methoxyestradiol with angiogenic and antiangiogenic factors. A, The positive correlation between maternal plasmatic 2ME levels and maternal serum levels of placental growth factor (PIGF) in the population with preeclampsia sample $(\mathrm{n}=50$; $r=.402$; $P=.004)$. B, The negative correlation between maternal plasmatic $2 M E$ levels and maternal serum levels of soluble fms-like tyrosine kinase $\mathrm{I}(\mathrm{sFlt})$ in the population with preeclampsia sample $(\mathrm{n}=50 ; r=-.325 ; P=.02 \mathrm{I})$. C and $\mathrm{D}$, The same correlations in the total population sample $(\mathrm{n}=\mathrm{II} 6 ; r=.436 ; P<.00 \mathrm{I}$ and $r=-.344 ; P<.00 \mathrm{I}$, respectively).

levels, it seems reasonable to suggest that 2ME may be a potential therapeutic agent. It has already been used in some clinical trials as a promising antitumoral agent, ${ }^{38}$ and the list of the tumor cell types and proliferative or inflammatory disorders that seem to respond to treatment with $2 \mathrm{ME}$ continues to increase. ${ }^{39,40}$ Furthermore, phase I and II clinical trials so far indicate that it is an estrogenic compound fairly well tolerated even at very high doses. ${ }^{39}$ The participation of $2 \mathrm{ME}$ in the pathogenesis of PE suggests that its supplementation might potentially prevent the development of the syndrome in susceptible pregnant women, opening a door toward a new therapeutic strategy for PE.

In summary, we have provided initial clinical evidence showing that $2 \mathrm{ME}$ levels are reduced in PE, and its low concentration is correlated with disease severity. This study adds additional support to the hypothesis that $2 \mathrm{ME}$ may be a key factor in maintaining angiogenic balance in the pathophysiology of PE, indicating that it could be used as a biomarker in the clinical management of the syndrome.

\section{Declaration of Conflicting Interests}

The author(s) declared no potential conflicts of interest with respect to the research, authorship, and/or publication of this article.

\section{Funding}

The author(s) received the following financial support for the research, authorship, and/or publication of this article: This work was supported by grants from the Ministerio de Ciencia e Innovación (BES200715929, BFU2009-11467) and Fundacion Séneca (08843/pi/08).

\section{References}

1. Ghulmiyyah L, Sibai B. Maternal mortality from preeclampsia/ eclampsia. Semin Perinatol. 2012;36(1):56-59.

2. Duley L. The global impact of pre-eclampsia and eclampsia. Semin Perinatol. 2009;33(3):130-137.

3. Shenoy V, Kanasaki K, Kalluri R. Pre-eclampsia: connecting angiogenic and metabolic pathways. Trends Endocrinol Metab. 2010;21(9):529-536.

4. Verlohren S, Stepan H, Dechend R. Angiogenic growth factors in the diagnosis and prediction of pre-eclampsia. Clin Sci (Lond). 2012;122(2):43-52.

5. Dubey RK, Jackson EK. Potential vascular actions of 2-methoxyestradiol. Trends Endocrinol Metab. 2009;20(8):374-379.

6. Berg D, Sonsalla R, Kuss E. Concentrations of 2-methoxyoestrogens in human serum measured by a heterologous immunoassay 
with an 125I-labelled ligand. Acta Endocrinol (Copenh). 1983; 103(2):282-288.

7. Berg FD, Kuss E. Serum concentration and urinary excretion of "classical" estrogens, catecholestrogens and 2-methoxyestrogens in normal human pregnancy. Arch Gynecol Obstet. 1992;251(1):17-27.

8. Lee SB, Wong AP, Kanasaki K, et al. Preeclampsia: 2-methoxyestradiol induces cytotrophoblast invasion and vascular development specifically under hypoxic conditions. Am J Pathol. 2010; 176(2):710-720.

9. Kanasaki K, Palmsten K, Sugimoto H, et al. Deficiency in catechol-O-methyltransferase and 2-methoxyoestradiol is associated with pre-eclampsia. Nature. 2008;453(7198):1117-1121.

10. Barchiesi F, Jackson EK, Fingerle J, Gillespie DG, Odermatt B, Dubey RK. 2-Methoxyestradiol, an estradiol metabolite, inhibits neointima formation and smooth muscle cell growth via double blockade of the cell cycle. Circ Res. 2006;99(3):266-274.

11. Bourghardt J, Bergström G, Krettek A, Sjöberg S, Borén J, Tivesten $\mathrm{A}$. The endogenous estradiol metabolite 2-methoxyestradiol reduces atherosclerotic lesion formation in female apolipoprotein E-deficient mice. Endocrinology. 2007;148(9):4128-4132.

12. Dubey RK, Jackson EK. Cardiovascular protective effects of 17beta-estradiol metabolites. J Appl Physiol. 2001;91(4):1868-1883.

13. Seeger H, Mueck AO, Lippert TH. Effect of estradiol metabolites on prostacyclin synthesis in human endothelial cell cultures. Life Sci. 1999;65(13):PL167-PL170.

14. Gui Y, Zheng XL, Zheng J, Walsh MP. Inhibition of rat aortic smooth muscle contraction by 2-methoxyestradiol. Am J Physiol Heart Circ Physiol. 2008;295(5):H1935-H1942.

15. Fenoy FJ, Hernandez ME, Hernandez M, Quesada T, Salom MG, Hernández I. Acute effects of 2-methoxyestradiol on endothelial aortic No release in male and ovariectomized female rats. Nitric Oxide. 2010;23(1):12-19.

16. Dubey RK, Tofovic SP, Jackson EK. Cardiovascular pharmacology of estradiol metabolites. J Pharmacol Exp Ther. 2004;308(2): 403-409.

17. Jobe SO, Tyler CT, Magness RR. Aberrant synthesis, metabolism, and plasma accumulation of circulating estrogens and estrogen metabolites in preeclampsia implications for vascular dysfunction. Hypertension. 2013;61(2):480-487.

18. Barnea ER, MacLusky NJ, DeCherney AH, Naftolin F. Catecholo-methyl transferase activity in the human term placenta. Am J Perinatol. 1988;5(2):121-127.

19. Lim JH, Kim SY, Kim DJ, et al. Genetic polymorphism of catechol-O-methyltransferase and cytochrome P450c17 $\alpha$ in preeclampsia. Pharmacogenet Genomics. 2010;20(10):605-610.

20. Hill LD, York TP, Kusanovic JP, et al. Epistasis between COMT and MTHFR in maternal-fetal dyads increases risk for preeclampsia. PLoS One. 2011;6(1):e16681.

21. Liang S, Liu X, Fan P, et al. Association between Val158Met functional polymorphism in the COMT gene and risk of preeclampsia in a Chinese population. Arch Med Res. 2012;43(2): 154-158.

22. Sata F, Yamada H, Suzuki K, et al. Functional maternal catecholO-methyltransferase polymorphism and fetal growth restriction. Pharmacogenet Genomics. 2006;16(11):775-781.
23. Ness RB, Sibai BM. Shared and disparate components of the pathophysiologies of fetal growth restriction and preeclampsia. Am J Obstet Gynecol. 2006;195(1):40-49.

24. Barnes CM, Mcelrath TF, Folkman J, Hansen AR. Correlation of 2-methoxyestradiol levels in cord blood and complications of prematurity. Pediatr Res. 2010;67(5):545-550.

25. Bonacasa B, Sanchez ML, Rodriguez F, et al. 2-Methoxyestradiol attenuates hypertension and coronary vascular remodeling in spontaneously hypertensive rats. Maturitas. 2008;61(4):310-316.

26. Hernandez M, Hernandez I, Rodríguez F, et al. Endothelial dysfunction in gestational hypertension induced by catechol-Omethyltransferase inhibition. Exp Physiol. 2013;98(3):856-866.

27. Glantzounis GK, Tsimoyiannis EC, Kappas AM, Galaris DA. Uric acid and oxidative stress. Curr Pharm Des. 2005;11(32): 4145-4151.

28. Berry CE, Hare JM. Xanthine oxidoreductase and cardiovascular disease: molecular mechanisms and pathophysiological implications. J Physiol. 2004;555(pt 3):589-606.

29. Hawkins TL, Roberts JM, Mangos GJ, Davis GK, Roberts LM, Brown MA. Plasma uric acid remains a marker of poor outcome in hypertensive pregnancy: a retrospective cohort study. BJOG. 2012;119(4):484-492.

30. Dubey RK, Gillespie DG, Keller PJ, Imthurn B, Zacharia LC, Jackson EK. Role of methoxyestradiols in the growth inhibitory effects of estradiol on human glomerular mesangial cells. Hypertension. 2002;39(2 pt 2):418-424.

31. Tofovic SP, Salah EM, Dubey RK, Melhem MF, Jackson EK. Estradiol metabolites attenuate renal and cardiovascular injury induced by chronic nitric oxide synthase inhibition. J Cardiovasc Pharmacol. 2005;46(1):25-35.

32. Hernández-Díaz S, Toh S, Cnattingius S. Risk of pre-eclampsia in first and subsequent pregnancies: prospective cohort study. BMJ. 2009;338:b2255.

33. Sohlberg S, Stephansson O, Cnattingius S, Wikström AK. Maternal body mass index, height, and risks of preeclampsia. Am J Hypertens. 2012;25(1):120-125.

34. England L, Zhang J. Smoking and risk of preeclampsia: a systematic review. Front Biosci. 2007;12:2471-2483.

35. Stejskalova L, Pavek P. The function of cytochrome P450 1A1 enzyme (CYP1A1) and aryl hydrocarbon receptor (AhR) in the placenta. Curr Pharm Biotechnol. 2011;12(5):715-730.

36. Schiettecatte J, Russcher H, Anckaert E, et al. Multicenter evaluation of the first automated Elecsys sFlt-1 and PlGF assays in normal pregnancies and preeclampsia. Clin Biochem. 2010;43(9):768-770.

37. Maynard S, Epstein FH, Karumanchi SA. Preeclampsia and angiogenic imbalance. Annu Rev Med. 2008;59:61-78.

38. Mooberry SL. New insights into 2-methoxyestradiol, a promising antiangiogenic and antitumor agent. Curr Opin Oncol. 2003; 15(6):425-430.

39. Mueck AO, Seeger H. 2-Methoxyestradiol-biology and mechanism of action. Steroids. 2010;75(10):625-631.

40. Machado-Linde F, Pelegrin P, Sanchez-Ferrer ML, Leon J, Cascales P, Parrilla JJ. 2-methoxyestradiol in the pathophysiology of endometriosis: focus on angiogenesis and therapeutic potential. Reprod Sci. 2012;19(10):1018-1029. 